\title{
Numerical Simulation of Optical Sensing by the Far Field Pattern Radiated by Periodic Grating Strips Over Silica Buffer on the Silicon Wire Waveguide
}

\author{
Andrei Tsarev ${ }^{1,2}\left(\mathbb{D}\right.$ and Vittorio M. N. Passaro ${ }^{3, *(1)}$ \\ 1 Laboratory of Optical Materials and Structures, Rzhanov Institute of Semiconductor Physics, SB RAS, \\ 630090 Novosibirsk, Russia; tsarev@isp.nsc.ru \\ 2 Laboratory of Semiconductor and Dielectric Materials, Physics Department, Novosibirsk State University, \\ 630090 Novosibirsk, Russia \\ 3 Photonics Research Group, Dipartimento di Ingegneria Elettrica e dell'Informazione, Politecnico di Bari, \\ Via E. Orabona 4, 70125 Bari, Italy \\ * Correspondence: vittorio.passaro@poliba.it; Tel.: +39-080-596-3850
}

Received: 31 August 2020; Accepted: 15 September 2020; Published: 16 September 2020

\begin{abstract}
This paper presents results of numerical modeling of a modified design of an optical sensor based on segmented periodic silicon oxynitride $(\mathrm{SiON})$ grating evanescently coupled with silicon wire. This segmented grating works as a leaky waveguide, which filters input power from a broadband optical source and radiates it as an outcoming optical beam with both a small wavelength band and a small beam divergence. The radiation angle strongly depends on the refractive index of the grating environment and provides sensor interrogation by measuring the far field pattern in the focal plane of the lens, which is placed near the sensor element. The device concept was verified by direct numerical modeling through the finite difference time domain (FDTD) method and provided moderate intrinsic limit of detection (iLOD) 0.004 RIU with a possible iLOD 0.001 RIU for $10 \mathrm{~mm}$-long structures.
\end{abstract}

Keywords: optical sensors; silicon wire; silicon oxynitride (SiON); segmented grating; far field pattern; numerical modeling; finite difference time domain (FDTD) method

\section{Introduction}

Publications on optical sensors cover about 20\% [1] of all publications registered by the Web of Science. These sensors utilize different arrangements [1-15] for reading signals. Among them, a lot of optical sensors use wavelength interrogation based on high precision measuring of the shift in resonance wavelength in the photonic structure, and the value depends on the environment of the sensor element [1-10]. Wavelength interrogation can be accomplished using a tunable laser with a fine linewidth or by launching the optical beam into the waveguide, which contains a broad wavelength spectrum, say, from the super-luminescence diode, and measuring the transmitting signal by the optical spectrum analyzer (OSA). Both these variants provide the possibility to develop advanced optical sensors [1-10] with the sensitivity depending on the design of the optical element and the spectrum resolution of the tunable laser or the OSA. Both readout schemes are rather costly, which prevents the wide spread of these sensor technologies. Alternative interrogation technology provides grating assisted sensors [13-15], which measures the far field pattern. as the radiation angle, due to Bragg conditions, is strongly dependent on optical wavelength, grating period, and environment index.

In the current paper, we present the numerical study of the modified variant of the far field pattern optical sensor with the segmented grating. The periodic segmented structure works both as the pass band filter, which selects part of the spectrum energy from the broadband input optical source, and as the grating coupler, which radiates power to be focused by the lens on the multi-element photonic 
sensor, which is used to measure the far field pattern. The previous variant of such a sensor [16] was based on the optical phenomena of "abnormal blocking" [17,18], which takes place in the silicon wire with the periodic segmented grating coupled by the evanescent field through the silica buffer. This design provides very high sensitivity ( $\sim 400 \mathrm{~nm} / \mathrm{RIU})$ but unfortunately has very low intrinsic limit of detection (iLOD) 0.01 RIU due to high propagation loss of the leaky waveguide formed by long-period segmented grating. The idea of this paper is to present the modified sensor design, which has both high sensitivity and moderate iLOD, which is useful for application as the liquid sensor works in water and has a high optical loss in the telecom band.

\section{Sensor Design}

The structure design, which is under investigation, is shown in Figure 1. The main reason for the high loss in the long-period grating leaky waveguide [17-19] is the radiative scattering of the diverge wave propagating in the free space of the environmental sensor between the segments. One can decrease this loss by decreasing the gap $\mathrm{D}(<200 \mathrm{~nm})$, but it provides some disadvantage due to effect of the partial gap filling by the liquid [20], which will course some degradation of the sensor performances.

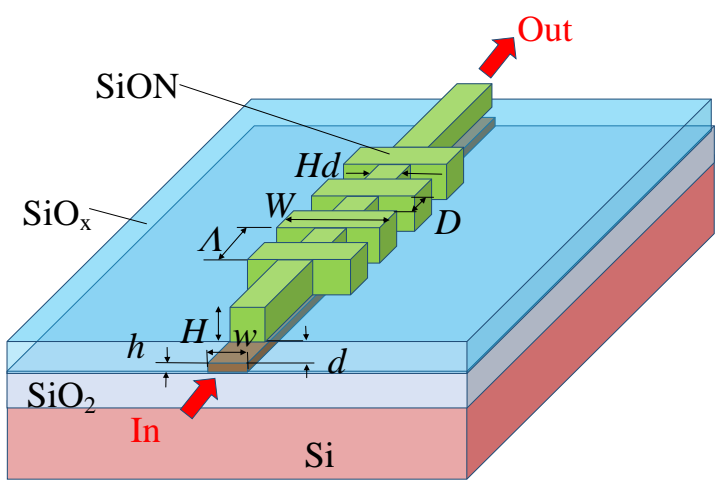

(a)

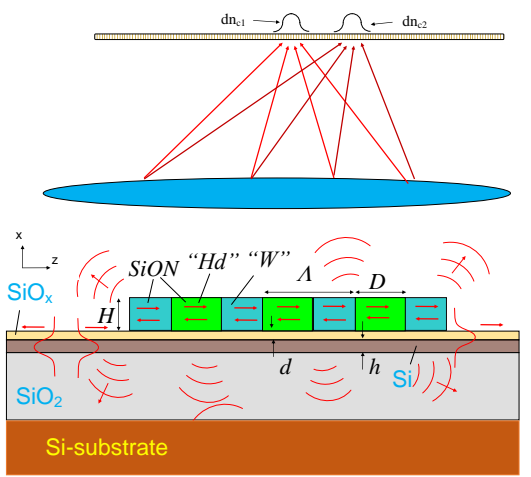

(b)

Figure 1. The scheme of the optical sensor based on the interaction of the guided wave with the virtual leaky wave of the segmented grating. (a) General view of segmented structure; (b) general arrangement

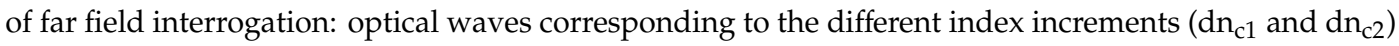
were focused at different positions on the photodetector screen.

We propose the use of segmented structures with a wide gap $(D>1000 \mathrm{~nm})$ by implementing the modified design that includes close-connected strips between the segments (see Figure 1a). The strip width $\mathrm{Hd}=450 \mathrm{~nm}$ was close to the fundamental mode cutoff. From one side, it did not cause significant degradation of sensitivity related to pure segmented grating, but from the other side, the optical wave that left the segmented part was guided forward up to the next segment and thus decreased the propagation loss in the structure.

Note that this design is very similar to the conventional grating filter, with the rectangular grating manufactured on the waveguide sides [21]. However, this grating filter was optimized for sensor application. Thus, contrary to the known design of the grating filter, this grating accomplished the two-stage process: at first, it provided coupling from the guide to the leaky modes, then this leaky wave radiated power by the same grating during the propagation along the structure. This radiated wave was focused by an additional lens on the multi-element detector, such as the CCD (charge coupled device) camera placed on the focal plane (see Figure 1b). This lens was placed over the flow chambers (not shown) and provided the possibility to measure and analyze the far field pattern variation under an index change of the liquid index under investigation.

The silicon wire had the height $\mathrm{h}=250 \mathrm{~nm}$ and the width $\mathrm{w}=450 \mathrm{~nm}$. It was placed on a thick $2 \mu \mathrm{m}$ silicon dioxide $\left(\mathrm{n}_{\mathrm{b}}=1.444\right)$ buffer. It corresponded to the optical waveguide, which supported the single quasi-TE (transverse electric)-polarized guided mode (electric field in the plane of the 
waveguide). The segmented structure was achieved by the periodic $(\Lambda=1.45 \mu \mathrm{m})$ strips from silicon oxynitride $(\mathrm{SiON})$, with the index at $\mathrm{n}_{\mathrm{p}}=1.80$. The desired value index of $\mathrm{SiON}$ can be given by the composition ratio $\mathrm{X}_{\mathrm{SiO} 2}=0.653$ of $\mathrm{SiO}_{2}$ in the $\mathrm{SiON}$ :

$$
\mathrm{n}_{\mathrm{pSiON}}=\mathrm{N}_{\mathrm{SiO} 2}+\mathrm{X}_{\mathrm{SiO} 2} \cdot\left(\mathrm{N}_{\mathrm{Si} 3 \mathrm{~N} 4}-\mathrm{N}_{\mathrm{SiO} 2}\right)
$$

The segmented grating strip length $\mathrm{D}_{\mathrm{s}}=\Lambda-\mathrm{D}$ was controlled by the period $\Lambda$ and the etching gap $\mathrm{D}$. We used the condition $\mathrm{D}=0.2 \mu \mathrm{m}$, which provided the compromise between the sensitivity and the propagation loss. The typical height and width were $\mathrm{H}=1.0 \mu \mathrm{m}$ and $\mathrm{W}=1.0 \mu \mathrm{m}$, respectively. Grating was separated from the silicon waveguide by a silica overlayer, with height $\mathrm{d}=400 \mathrm{~nm}$ and index $\mathrm{n}_{\mathrm{S}}=1.4$.

In the current sensor design, the guided optical beam, which contained a broad spectral range, arrived from the left into the grating area (see Figure 1a). The presence of the periodic grating provided resonance-type interaction (at optical wavelength $\lambda_{\mathrm{m}}$ ) of the guided mode in the silicon wire (effective index $\mathrm{N}_{\mathrm{g}}$ ) and the leaky mode (effective index $\mathrm{N}_{\mathrm{L}}$ ), propagated in the segmented SiON structure. The full width at half-maximum (FWHM) of the grating resonance $\Delta \lambda$ was mainly limited by the structure length and propagation loss of the leaky wave and typically was in the range of 1-5 nm. This filtered part of the input spectrum was coupled into the segmented structure and then was radiated along the propagation. The radiated angle of the maximum intensity at optical wavelength $\lambda_{\mathrm{m}}$ was described by the Bragg condition and provided information about the index of the environment $n_{c}$.

The fabrication of such structures could be carried out by using a silicon photonic process developed within the conventional CMOS (complementary metal oxide semiconductor) foundry. For example, one can use the technology already used to manufacture a spot-size converter (SSC) with a $\mathrm{SiO}_{2}$ spacer layer between tapered $\mathrm{Si}$ and $\mathrm{SiON}$ waveguides for fiber-to-chip coupling [22]. They deposited $\mathrm{SiO}_{2}$ and $\mathrm{SiON}$ with a low refractive index at a low temperature by the PECVD (plasma enhanced chemical vapor deposition) method. For the SSC with the $\mathrm{SiO}_{2}$ spacer, the $\mathrm{SiO}_{2}(\mathrm{n}=1.45)$ spacer layer was deposited on the $\mathrm{Si}$ wire waveguide at $350{ }^{\circ} \mathrm{C}$, followed by deposition of a 1.2 - $\mu \mathrm{m}$-thick $\mathrm{SiON}\left(\mathrm{n}=1.62\right.$ ) film from a gas mixture of $\mathrm{N}_{2} \mathrm{O}, \mathrm{NH}_{3}$, and $\mathrm{SiH}_{4}$ at $300{ }^{\circ} \mathrm{C}$. A $1.2-\mu \mathrm{m}$-square $\mathrm{SiON}$ waveguide with vertical walls was fabricated with an i-line stepper and by dry etching with $\mathrm{CHF}_{3}$ (trifluoromethane). It must be noted that the fabricated $\mathrm{SiON}$ waveguide cores are somewhat distorted and are related to the rectangular shape as the thin $\mathrm{SiO}_{2}$ layer is not planarized when it covers the silicon wire. That is not important for SSC, but in our modeling, we assumed that structures with a planarized $\mathrm{SiO}_{2}$ spacer were inserted between the $\mathrm{Si}$ wire waveguide and the $\mathrm{SiON}$ secondary waveguide. The flat structures could be manufactured by using flowable oxide $(n=1.40)$ or by introducing the lift-off process used for the planarization of the waveguide surface similar to technology described in [23], which used $\mathrm{Cr}$ as the mask for the etching of the core and the cladding materials for $\mathrm{TiO}_{2} / \mathrm{Ta}_{2} \mathrm{O}_{5} / \mathrm{SiO}_{2}$ structure. In our case, the $\mathrm{Cr}$ mask could be used to develop the silicon wire. $\mathrm{A} \mathrm{SiO}_{2}$ thin layer (equal to the height of the silicon wire) could then be deposited by the PECVD method over the silicon wire waveguide. Next, the $\mathrm{SiO}_{2}$ layer on the $\mathrm{Cr}$ mask was lifted off by wet etching of $\mathrm{Cr}$. Thus, a flat surface was obtained. After planarization of waveguide surface, one can follow all of the technology process used to manufacture a spot-size converter [22], with the only difference found in the mask shape for the SiON waveguide, which now contains the grating strips.

\section{Numerical Modeling}

This structure is investigated by numerical modeling, which has been performed using the finite difference time domain (FDTD) method, which has proved its reliability many times for such a task [24]. For simulation, we utilized the commercial software package FullWave by RSoft-SYNOPSYS [25]. In order to examine the long structures, we utilized FDTD under two-dimensional (2D) approximation based on the effective index method (EIM) [26]. 
As we have discussed before, the EIM has a fundamental limitation $[27,28]$, which takes place when trying to analyze the pulsed excitation of waveguide structures by the 2D FDTD method. The reason is that, in this case, the two-dimensional EIM approximation does not take into account the waveguide dispersion and thus considers the wrong value of the group index [27], which determines both the sensitivity and the linewidth. The typical error could be as much as $40 \%$ or more [27].

We propose the dispersion compensated algorithm [17] to fix this error problem. The presence of waveguide dispersion leads to the fact that the resonance wavelength $\lambda_{\mathrm{m}}$, which is determined by the 2D FDTD modeling, depends on the center wavelength $\lambda_{0}$, at which the impulse excitation and the spectrum analysis of the waveguide structure is carried out. It was shown [17] that the correct drop wavelength could be found from the condition $\lambda_{\mathrm{m}}\left(\lambda_{0}\right)=\lambda_{0}$, which provides $\lambda_{\mathrm{m}}$ to be exactly equal to the desired condition obtained for the monochromatic excitation of a waveguide. As a result of this description, the correct wavelength responses at any desired $\mathrm{dn}_{\mathrm{c}}$ corresponded to those simulations, when $\lambda_{\mathrm{m}}\left(\lambda_{0}\right)=\lambda_{0}$. For the simplicity, we determined two basic wavelengths $\lambda_{\mathrm{m}}$ for two cases, $\mathrm{dn}_{\mathrm{c}}=0$ and $\mathrm{dn}_{\mathrm{c}}=0.01$. We then implemented the linear interpolation for the wavelength coordinate:

$$
\lambda_{\mathrm{c}}=\mathrm{A}+\mathrm{B} \cdot \lambda_{0}
$$

where $\mathrm{A}=446.4 \mathrm{~nm}$ and $\mathrm{B}=0.7112$, which are obtained from these two basic wavelength simulations. Thus, all wavelength responses of the transmitting power to the leaky wave $T_{2}\left(\lambda_{c}\right)$ will now correctly take into account the waveguide dispersion, which provides much more accuracy in the determination of all sensor parameters, including the iLOD and sensitivity $\mathrm{S}_{\mathrm{n}}$. We called this algorithm the dispersion compensated effective index method (DCEIM). One must note that this method can be applied for any numerical tasks that implement the 2D FDTD method to examine the impulse propagation in the waveguide structures.

Using this dispersion compensated algorithm, we found, using the 2D FDTD method, the sensitivity $\mathrm{S}_{\mathrm{n}}=\partial \lambda / \partial \mathrm{n}_{\mathrm{c}}=340 \mathrm{~nm} / \mathrm{RIU}$ (refractive index unit) for the wavelength interrogation. Note that the similar modeling with traditional EIM provides strongly incorrect values for the sensitivity $S_{n}=480 \mathrm{~nm} / \mathrm{RIU}$.

As mentioned before, the sensor element based on the segmented grating over the silicon waveguide can implement different readout schemes. The application of the DCEIM to traditional wavelength interrogation is illustrated in Figure 2a. This application is typical for a lot of sensors but it requires tunable lasers or a high resolution OSA. For the far field interrogation, it is possible to measure the cross-field distribution on the focal plane of the lens (see Figure 2b). To improve the accuracy, we also implemented the DCEIM algorithm, which, in our case, included the linear transformation of the coordinate according to the relation:

$$
\mathrm{X}_{\mathrm{c}}=\mathrm{A}_{\mathrm{x}}+\mathrm{B}_{\mathrm{x}} \cdot \mathrm{X}
$$

where $A_{x}=156.5$ and $B_{x}=0.948$, which are obtained from linear interpolation of the simulation results for $\mathrm{dn}_{\mathrm{c}}=0$ and $\mathrm{dn}_{\mathrm{c}}=0.01$.

For the maximum number of segments $(M=1024)$, which were possible to simulate in our case, we got almost the same value of intrinsic limit of detection for both the wavelength interrogation $\left(\mathrm{iLOD}_{\mathrm{W}}=\delta \mathrm{n} \cdot \Delta \lambda / \Delta \lambda_{\mathrm{FWHM}}=0.0038 \mathrm{RIU}\right)$ and the far field interrogation $\left(\mathrm{iLOD}_{\mathrm{FF}}=\delta \mathrm{n} \cdot \Delta \mathrm{X} / \Delta \mathrm{X}_{\mathrm{FWHM}}=\right.$ 0.0033 RIU). Here, for $\Delta \lambda$ and $\Delta \mathrm{X}$, the shift of the peaks position for the index increment $\delta n, \Delta \lambda_{\mathrm{FWHM}}$, and $\Delta \mathrm{X}_{\mathrm{FWHM}}$ were the relative full width at half-maximum.

The extremum positions, $\lambda_{m}$ and $X_{m}$, as a function of the different index variations $\mathrm{dn}_{\mathrm{c}}$ of the environment (water) for the wavelength and far field interrogation, respectively, are shown on Figure 3. One can see their linear dependences on $\mathrm{dn}_{\mathrm{c}}$ with the constant slope, which describes the sensitivity of the sensor. For example, the dispersion compensated algorithm provides the sensitivity $\mathrm{S}_{\mathrm{n}}=\partial \lambda / \partial \mathrm{n}_{\mathrm{c}}=340 \mathrm{~nm} / \mathrm{RIU}$ for the wavelength interrogation. However, we have to highlight that similar modeling under the traditional EIM provides a strongly incorrect value for the sensitivity $\mathrm{S}_{\mathrm{n}}=480 \mathrm{~nm} / \mathrm{RIU}$. 


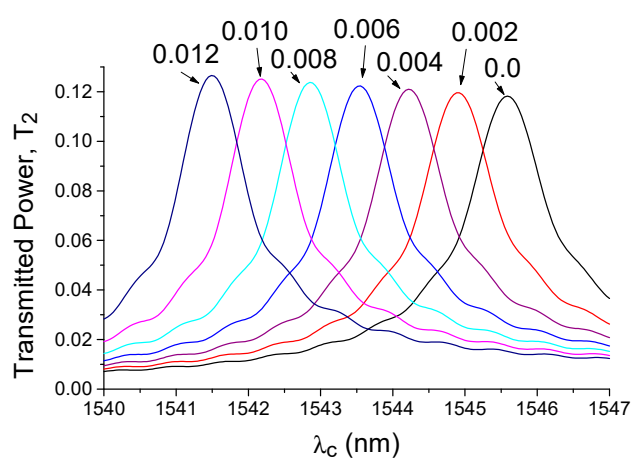

(a)

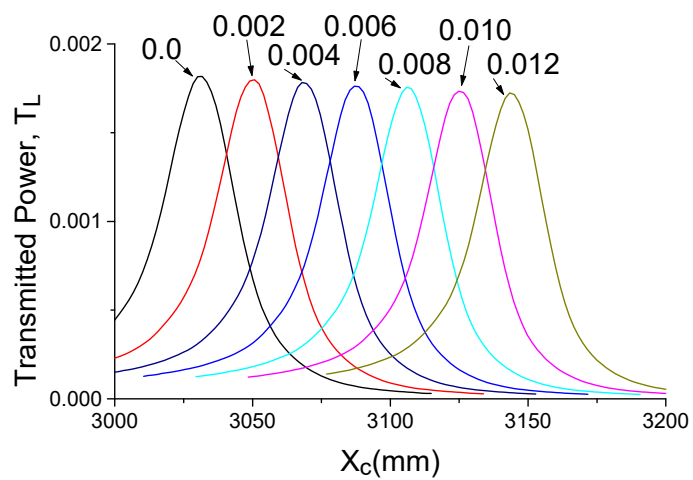

(b)

Figure 2. Simulated characteristics of the optical sensors and different index variations $\mathrm{dn}_{\mathrm{c}}$ of the environment (water). (a) Transmitting power $\left(\mathrm{T}_{2}\right)$ relative to the input signal of the leaky wave propagated in segmented grating; (b) the total power $\left(\mathrm{T}_{\mathrm{L}}\right.$ ) distribution (as a sum of 100 different wavelengths) in the focal plane of the ideal lens with $10 \mathrm{~mm}$ focal length. Simulation by the two-dimensional (2D) finite difference time domain (FDTD) under the dispersion compensated the effective index method approximation, which included the linear transformation of the coordinates (2) and (3).

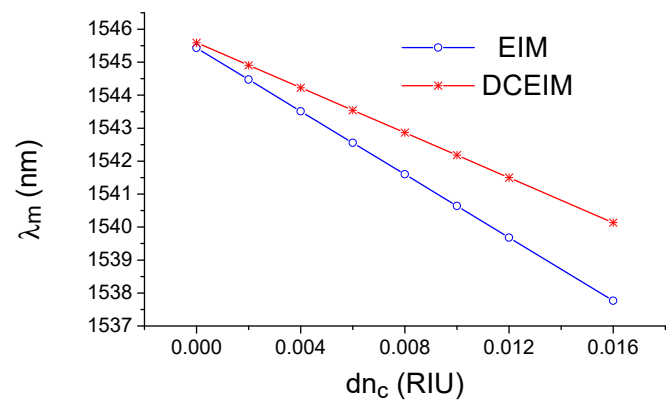

(a)

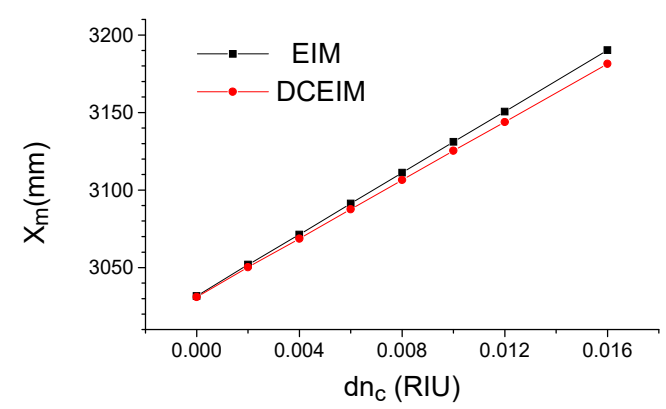

(b)

Figure 3. Simulated characteristics of the optical sensors obtained under the effective index method (EIM) and the dispersion compensated effective index method (DCEIM) approximations for the different index variations $\mathrm{dn}_{\mathrm{c}}$ of the environment (water). (a) Wavelength position $\lambda_{\mathrm{m}}$ of the maximum transmitting power to the leaky wave propagated in segmented grating; $(\mathbf{b})$ the coordinate position $X_{m}$ of the maximum transmitting power in the focal plane of the ideal lens with $10 \mathrm{~mm}$ focal length. Simulation by the 2D FDTD.

It must be noted that by increasing the grating length $\left(\mathrm{Lg}_{\mathrm{g}}=\Lambda \times \mathrm{M}\right)$, one can increase, to some extent, the wavelength resolution, but soon it will come to the maximum value restricted by the loss of the leaky way. For example, a long sensor will reach iLOD $\sim 1.5 \times 10^{-3} \mathrm{RIU}\left(\mathrm{Lg}_{\mathrm{g}}=5 \mathrm{~mm}\right)$ and iLOD $\sim 1 \times 10^{-3}$ RIU $\left(\mathrm{Lg}_{\mathrm{g}}=10 \mathrm{~mm}\right)$. We estimated the minimum possible iLOD by analyzing the short structure $(\mathrm{M}=3)$ by using the periodic boundary conditions. It was found that the minimum iLOD for our design was around $7.6 \times 10^{-4}$ RIU and it occurred due to the high optical loss in water at the optical wavelength $(1550 \mathrm{~nm})$.

Note that the measuring arrangement was then much cheaper than for the case of the optical spectrum analyzer (OSA) or the tunable laser. The experimental limit of detection (LOD) depends on the experimental setup, integration time, temperature fluctuations, and the noise of the system, which is limited by intensity fluctuations [29]. Typically, it is possible to measure the wavelength position with an accuracy of about 1/15 of the FWHM [30]. Thus, we got the experimental LOD = iLOD/15 $<0.0001$ RIU. For the case of the far field interrogation, the fluctuations of the intensity at different wavelengths had to be averaged within the angle divergence of the monochromatic wave. The pattern distribution 
measured at the focal plane of the lens (see Figure $2 b$ ) was much smoother than the relative wavelength response measured by the OSA. As the smaller intensity fluctuations were important for decreasing the LOD [29], thus the experimental LOD of our far field interrogation design had to be much smaller than for the wavelength interrogation, with a broad optical source of the same structure. This makes it possible to determine a small variation of the refractive index (below 0.0001 RIU) by a very simple and cheap interrogation scheme.

Note that, in our design, we proposed to use a cheap broadband optical source instead of a costly tunable laser or OSA. Part of the input energy spectrum was selected by the grating coupler, which was further diffracted by the same grating. The angle divergence, when projected on the focal plane of the lens, was always larger than the monochromatic illumination of the same structure by the tunable laser with a small line width (as with the conventional grating sensor [13].) Thus, in any case, the experimental LOD of the proposed sensor is larger than the competitor that utilizes the tunable laser with a linewidth smaller than the sensor linewidth. Our design by the expense of some degradation of the sensitivity is intended to use the cheaper components (say, no tunable laser or OSA) and to provide the possibility of broad use of it as a liquid type of sensor, where the experimental LOD (due to high optical loss in water) is several orders larger than for the case of gas sensors used by other structures [31], such as photonic crystals [32] or phase-shifted Bragg gratings [33] with super high quality factors.

\section{Conclusions}

We have described and carried out numerical modeling of a modified type of optical sensor with far field interrogation, which utilizes the segmented periodic structure with narrow connected strips placed over silica buffer on the silicon wire. The tunnel coupling of the guided wave to the segmented structure provides excitation of the leaky wave in the periodic segmented grating. The latter radiates power as an outgoing optical beam. This process has a resonance nature and takes place at a small wavelength range (around $1 \mathrm{~nm}$ ), thus the outgoing optical beam has a small divergence, which makes it possible to determine small index variations of environmental liquid by measuring the shift in the far field pattern position. This can be accomplished by commercial CCD arrays placed in the focal plane of the lens.

The sensor resolution is quite high (iLOD $=0.001-0.004 \mathrm{~nm} / \mathrm{RIU}$ ), and, by the internal smoothing of the radiated pattern, one can get the experimental LOD smaller than 0.0001 RIU. Additionally, the sensor design is simpler and cheaper than other state-of-the-art optical solutions, where the sensor resolution depends on a more expensive design based on the use of the tunable laser or OSA.

Author Contributions: The conceptualization of this work was made by both authors. A.T. carried out investigation and formal analysis, A.T. and V.M.N.P. made the writing-review and editing. All authors have read and agreed to the published version of the manuscript.

Funding: This research received no external funding.

Acknowledgments: The authors are very thankful to SYNOPSYS for providing RSoft software for FDTD simulations.

Conflicts of Interest: The authors declare no conflict of interest.

\section{References}

1. Tsarev, A. Overview of integrated optical sensors based on silicon-Forecasts and results of the decade. (Invited paper). Optoelectron. Instrum. Data Process. 2020, in press. Russian Text published in Avtometriya 2020, 56, 111-133.

2. Fernández Gavela, A.; Grajales García, D.; Ramirez, J.C.; Lechuga, L.M. Last advances in silicon-based optical biosensors. Sensors 2016, 16, 285. [CrossRef]

3. Steglich, P.; Hülsemann, M.; Dietzel, B.; Mai, A. Optical Biosensors Based on Silicon-On-Insulator Ring Resonators: A Review. Molecules 2019, 24, 519. [CrossRef] [PubMed] 
4. Luan, E.; Shoman, H.; Ratner, D.M.; Cheung, K.C.; Chrostowski, L. Silicon Photonic Biosensors Using Label-Free Detection. Sensors 2018, 18, 3519, Erratum in 2019, 19, 1161. [CrossRef]

5. Xu, Y.; Hu, X.; Kundu, S.; Nag, A.; Afsarimanesh, N.; Sapra, S.; Mukhopadhyay, S.C.; Han, T. Silicon-Based Sensors for Biomedical Applications: A Review. Sensors 2019, 19, 2908. [CrossRef] [PubMed]

6. Angelopoulou, M.; Kakabakos, S.; Petrou, P. Label-Free Biosensors Based onto Monolithically Integrated onto Silicon Optical Transducers. Chemosensors 2018, 6, 52. [CrossRef]

7. Arshavsky-Graham, S.; Massad-Ivanir, N.; Segal, E.; Weiss, S. Porous silicon-based photonic biosensors: Current status and emerging applications. Anal. Chem. 2018, 91, 441-467. [CrossRef]

8. Ghosh, S.; Dar, T.; Viphavakit, C.; Pan, C.; Kejalakshmy, N.; Rahman, B.M.A. Compact Photonic SOI Sensors. In Computational Photonic Sensors; Hameed, M., Obayya, S., Eds.; Springer: Cham, Switzerland, 2019; pp. 343-383.

9. Chen, Y.; Liu, J.; Yang, Z.; Wilkinson, J.S.; Zhou, X. Optical biosensors based on refractometric sensing schemes: A review. Biosens. Bioelectron. 2019, 144, 111693. [CrossRef]

10. Luan, E.; Yun, H.; Laplatine, L.; Dattner, Y.; Ratner, D.M.; Cheung, K.C.; Chrostowski, L. Enhanced sensitivity of subwavelength multibox waveguide microring resonator label-free biosensors. IEEE J. Sel. Top. Quantum Electron. 2019, 25, 7300211. [CrossRef]

11. Mariani, S.; Strambini, L.M.; Paghi, A.; Barillaro, G. Low-Concentration Ethanol Vapor Sensing with Nanostructured Porous Silicon Interferometers Using Interferogram Average over Wavelength Reflectance Spectroscopy. IEEE Sens. J. 2018, 18, 7842-7849. [CrossRef]

12. Lova, P.; Manfredi, G.; Bastianini, C.; Mennucci, C.; Buatier de Mongeot, F.; Servida, A.; Comoretto, D. Flory-Huggins Photonic Sensors for the Optical Assessment of Molecular Diffusion Coefficients in Polymers. ACS Appl. Mater. Interfaces 2019, 11, 16872-16880. [CrossRef] [PubMed]

13. Cottier, K.; Wiki, M.; Voirin, G.; Gao, H.; Kunz, R.E. Label-free highly sensitive detection of (small) molecules by wavelength interrogation of integrated optical chips. Sens. Actuators B Chem. 2003, 91, 241-251. [CrossRef]

14. Darwish, N.; Caballero, D.; Moreno, M.; Errachid, A.; Samitier, J. Multi-analytic grating coupler biosensor for differential binding analysis. Sens. Actuators B Chem. 2010, 144, 413-417. [CrossRef]

15. Triggs, G.J.; Wang, Y.; Reardon, C.P.; Fischer, M.; Evans, G.J.; Krauss, T.F. Chirped guided-mode resonance biosensor. Optica 2017, 4, 229-234. [CrossRef]

16. Passaro, V.M.N.; De Leonardis, F.; Tsarev, A.; Kolosovsky, E. Concept of Optical Sensor Utilising the Far Field Pattern Radiated by Periodic Grating Strips Over Silica Cladding on the Silicon Wire Waveguide. In Proceedings of the Ninth International Conference on Sensor Device Technologies and Applications, Venice, Italy, 16-20 September 2018; pp. 28-31, Best paper Award.

17. Tsarev, A.; Kolosovsky, E.; De Leonardis, F.; Passaro, V.M.N. Numerical Simulation of a Novel Sensing Approach Based on Abnormal Blocking by Periodic Grating Strips near the Silicon Wire Waveguide. Sensors 2018, 18, 1707. [CrossRef]

18. Tsarev, A.; De Leonardis, F.; Passaro, V.M.N. Numerical Modeling of Abnormal Blocking Effect for the Design of Novel Optical Sensor Element Constructed by Periodic Grating Strips Over $\mathrm{Si} / \mathrm{SiO}_{2}$ Wire Waveguide. Phys. Status Solidi. A 2019, 216, 1800480. [CrossRef]

19. Kolosovskii, E.A.; Tsarev, A.V. Abnormal blocking of a guided mode propagating in a silicon optical waveguide with periodic tunnel coupling. Quantum Electron. 2017, 47, 58-64. [CrossRef]

20. Claes, T.; Girones Molera, J.; De Vos, K.; Schacht, E.; Baets, R.; Bienstman, P. Label-Free Biosensing With a Slot-Waveguide-Based Ring Resonator in Silicon on Insulator. IEEE Photonics J. 2009, 1, 197-204. [CrossRef]

21. Yen, T.-H.; Hung, Y.-J. Narrowband dual-wavelength silicon waveguide Bragg reflectors. J. Light. Technol. 2019, 37, 5326-5332. [CrossRef]

22. Maegami, Y.; Takei, R.; Omoda, E.; Amano, T.; Okano, M.; Mori, M.; Kamei, T.; Sakakibara, Y. Spot-size converter with a $\mathrm{SiO}_{2}$ spacer layer between tapered $\mathrm{Si}$ and $\mathrm{SiON}$ waveguides for fiber-to-chip coupling. Opt. Express 2015, 23, 21287-21295. [CrossRef]

23. Sekimoto, T.; Ikuta, S.; Pan, W.; Chu, S.T.; Kokubun, Y. Vertical antiresonant reflecting optical waveguide coupler for three-dimensional optical interconnects: Optimum design for large tolerance, high coupling efficiency, and short coupling length. Appl. Opt. 2000, 39, 426-430. [CrossRef] [PubMed]

24. Vaskevicius, K.; Gabalis, M.; Urbonas, D.; Balcytis, A.; Petruskevicius, R.; Juodkazis, S. Enhanced sensitivity and measurement range SOI microring resonator with integrated one-dimensional photonic crystal. J. Opt. Soc. Am. B 2017, 34, 750-755. [CrossRef] 
25. Rsoft FullWave by SYNOPSYS. Photonic Design Software. Available online: https://www.synopsys.com/ optical-solutions/rsoft.html (accessed on 18 January 2018).

26. Chiang, K.S. Dual effective-index method for the analysis of rectangular dielectric waveguides. Appl. Opt. 1986, 25, 2169-2174. [CrossRef] [PubMed]

27. Tsarev, A. Modified effective index method to fit the phase and group index of 3D photonic wire waveguide. Opt. Lett. 2013, 38, 293-295. [CrossRef]

28. O'Faolain, L.; Tsarev, A. Experimental demonstration of original optical filter based on multiply coupled waveguides. Opt. Lett. 2014, 39, 3627-3629. [CrossRef]

29. Hu, J.; Sun, X.; Agarwal, A.; Kimerling, L.C. Design guidelines for optical resonator biochemical sensors. JOSA B. 2009, 26, 1032-1041. [CrossRef]

30. Vos, K.; Bartolozzi, I.; Schacht, E.; Bienstman, P.; Baets, R. Silicon-on-insulator microring resonator for sensitive and label-free biosensing. Opt. Express 2007, 15, 7610-7615. [CrossRef]

31. Xu, Y.; Bai, P.; Zhou, X.; Akimov, Y.; Png, C.E.; Ang, L.K.; Knoll, W.; Wu, L. Optical Refractive Index Sensors with Plasmonic and Photonic Structures: Promising and Inconvenient Truth. Adv. Opt. Mater. 2019, 7, 1801433. [CrossRef]

32. Pitruzzello, G.; Krauss, T.F. Photonic crystal resonances for sensing and imaging. J. Opt. 2018, 20, 073004. [CrossRef]

33. Wang, X.; Flueckiger, J.; Schmidt, S.; Grist, S.; Fard, S.T.; Kirk, J.; Doerfler, M.; Cheung, K.C.; Ratner, D.M.; Chrostowski, L. A silicon photonic biosensor using phase-shifted Bragg gratings in slot waveguide. J. Biophotonics 2013, 6, 821-828. [CrossRef]

(C) 2020 by the authors. Licensee MDPI, Basel, Switzerland. This article is an open access article distributed under the terms and conditions of the Creative Commons Attribution (CC BY) license (http://creativecommons.org/licenses/by/4.0/). 